\title{
Evidence that a single EF-Ts suffices for the recycling of multiple and divergent EF-Tu species in Streptomyces coelicolor A3(2) and Streptomyces ramocissimus
}

\author{
Gertjan Hoogvliet, Gilles P. van Wezel and Barend Kraal \\ Author for correspondence: Barend Kraal. Tel: +31 71 5274770. Fax: +31 715274340 . \\ e-mail:B.kraal@chem.leidenuniv.nl
}

Department of Biochemistry, Leiden Institute of Chemistry, Leiden University, PO Box 9502, 2300 RA Leiden, The Netherlands
The tsf genes from Streptomyces coelicolor A3(2) and Streptomyces ramocissimus, encoding the guanine-nucleotide exchange factor EF-Ts, were cloned and sequenced. Streptomycetes have multiple and highly divergent EFTu species, with EF-Tu1 and EF-Tu3 showing only about $65 \%$ amino acid sequence identity, and yet these can apparently interact with a single EF-Ts species. tsf lies in an operon with rpsB, which encodes ribosomal protein S2. The amino acid sequence of $S 2$ from $S$. coelicolor differs from most other bacterial S2 homologues in having a C-terminal extension of $\mathbf{7 0}$ aa residues with a highly repetitive organization, the function of which is unknown. Transcription analysis of the rpsB-tsf operon of $S$. coelicolor by promoter probing, nuclease $\mathrm{S} 1$ mapping and Northern blotting revealed that the genes give rise to a bicistronic transcript from a single promoter upstream of rpsB. An attenuator was identified in the rpsB-tsf intergenic region; it results in an approximately $2: 1$ ratio of rpsB vs tsf transcripts. Although tuf1, encoding the major EF-Tu, is located in the rpsL ribosomal protein operon, an additional promoter in the fus-tuf1 intergenic region leads to a significant excess of EFTu over ribosomes. Most amino acid residues known from the Escherichia coli crystal structure of the EF-Tu.EF-Ts complex to be directly involved in interaction between the two elongation factors are conserved between $E$. coli and Streptomyces. However, whenever interaction residues in the EF-Tu moiety show divergence among Streptomyces EF-Tu1, EF-Tu2 and EF-Tu3, the single Streptomyces EF-Ts exhibits compensatory substitutions of the corresponding residues. These apparently enable productive interaction to occur with all three EF-Tus.

Keywords : $r p s B$ operon, $t s f$ regulation, GTPase switch protein, guanine-nucleotide exchange factor, ribosomal protein S2

\section{INTRODUCTION}

Elongation factor (EF)-Tu plays a pivotal role in the prokaryotic elongation cycle (for a review see Krab \& Parmeggiani, 1998). In its active conformation, EF-Tu transfers aminoacyl-tRNA to the ribosomal A-site. After initial recognition, correct codon-anticodon interaction

Abbreviation: EF, elongation factor.

The GenBank accession numbers for the sequences of the $S$. coelicolor rpsB-tsf operon and the $S$. ramocissimus tsf gene determined in this work are AF034101 and AF130345, respectively. triggers activity at the GTPase centre of EF-Tu, which leads to aminoacyl-tRNA proof-reading and to dissociation of EF-Tu.GDP, followed by peptide-chain elongation at the peptidyl transferase centre of the ribosome, and translocation by EF-G. The released EF$\mathrm{Tu} \cdot \mathrm{GDP}$ is subsequently regenerated to its GTP-bound form by the action of the nucleotide-exchange factor EFTs. EF-Tu represents one of the best-studied members of the superfamily of GTPase switch proteins (Bourne et al., 1991; Kraal et al., 1993).

In Escherichia coli, EF-Tu is the most abundant cytoplasmic protein and occurs at a 5- to 10-fold molar 
excess over ribosomes. It is encoded by two tuf genes, tuf $A$ and $t u f B$, with their gene products differing only in the C-terminal amino acid residue. tufA is located in the rpsL operon, immediately downstream of fus, the gene for EF-G, which in turn is preceded by the ribosomal protein genes $r p s L$ and $r p s G$ (Lindahl \& Zengel, 1986). tufB is the distal gene in an operon with four tRNA genes (Lee et al., 1981; Miyajima et al., 1981). Consequently, while transcription of tufA is subject to the same regulatory controls as ribosomal protein genes (Jinks-Robertson \& Nomura, 1987), tufB transcription is regulated in the same way as stable RNA genes (van Delft et al., 1987). Nevertheless, expression of tufA and tufB is coordinately regulated in E. coli, as shown by the almost constant $1: 1$ ratio of EF-TuA and EF-TuB across a range of growth rates (van der Meide et al., 1982). Similar to fus and tufA, the single gene for EF-Ts in E. coli, tsf, is also located in a ribosomal protein operon, $r p s B-t s f$, which forms a single transcription unit.

The three-dimensional structure of the E. coli EF$\mathrm{Tu} \cdot \mathrm{EF}-\mathrm{Ts}$ complex was determined to a resolution of $2.5 \AA$ (Kawashima et al., 1996). From the crystal structure it appears that domain I of EF-Tu (encompassing the first $200 \mathrm{aa}$, including the guaninenucleotide-binding site) interacts predominantly with the N-terminal half of EF-Ts, while EF-Tu domain III (encompassing the last $100 \mathrm{aa}$ ) interacts with the Cterminal part of the EF-Ts core (i.e. residues 140-180 and 230-260). Mutational analysis of EF-Ts confirmed that residues in the N-terminal domain and in subdomain $\mathrm{C}$ of the EF-Ts core are crucial for this interaction (Zhang et al., 1998). The driving force behind GDP release from EF-Tu is probably the dislocation of $\mathrm{Mg}^{2+}$ from the molecule, which is catalysed by EF-Ts (Kawashima et al., 1996).

In most Gram-positive bacteria, the high concentration of EF-Tu in the cytoplasm is maintained by a single tuf gene. Interestingly, multiple tuf genes are found in members of the genus Streptomyces, a Gram-positive soil bacterium with a complex mycelial life cycle that includes sporulation (Hopwood et al., 1995). For example, three tuf genes occur in Streptomyces ramocissimus, the producer of kirromycin, an antibiotic that binds specifically to EF-Tu. The $S$. ramocissimus tuf genes, designated $t u f 1, t u f 2$ and $t u f 3$, encode EF-Tus that are surprisingly heterogeneous: EF-Tu3 shows only about $65 \%$ amino acid identity with EF-Tu1 and EFTu2 (Vijgenboom et al., 1994). Streptomyces coelicolor A3(2) and other streptomycetes contain two tuf genes (van Wezel, 1994), designated tuf1 and tuf3 by reference to the corresponding genes in S. ramocissimus. Streptomyces tuf 1 encodes the major EF-Tu and, like E. coli tuf $A$, is located in the rpsL operon; it is transcribed at a very high level during exponential growth (van Wezel et al., 1995) from the rpsL operon promoter and a promoter specific for tuf1 (Tieleman et al., 1997). The roles of $t u f 2$ and $t u f 3$ are unclear; under normal growth conditions, the gene products could not be detected. Interestingly, tuf 3 transcription was shown to be subject to positive stringent control, and is also induced by other stress conditions, including heat shock (van Wezel et al., 1995; G. P. van Wezel, unpublished results).

The multiplicity of tuf genes in various Streptomyces species, and the high level of divergence between the gene products, raised the question as to whether the latter interact with a single EF-Ts species. This prompted investigation into the number of $t s f$ genes in streptomycetes and the homology of the gene products to other known EF-Ts species. In this paper we present the sequences of the single $t$ sf genes in S. coelicolor A3(2) and $S$. ramocissimus, and provide a transcriptional analysis of the $S$. coelicolor rpsB-tsf operon both in vivo and in vitro.

\section{METHODS}

Bacterial strains, culture conditions and plasmids. S. coelicolor A3(2) strains M145 (prototrophic, SCP1- SCP2 $^{-}$;

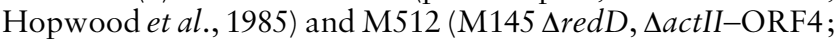
Floriano \& Bibb, 1996) were obtained from the John Innes Centre strain collection and used for transformation and propagation of Streptomyces plasmids. Protoplast preparation and transformation were performed as described by Hopwood et al. (1985). SFM medium (mannitol, $20 \mathrm{~g} \mathrm{l}^{-1}$; soya flour, $20 \mathrm{~g}$ $\mathrm{l}^{-1}$; agar, $20 \mathrm{~g} \mathrm{l}^{-1}$; dissolved in tap water) is a modified version of that reported by Hobbs et al. (1989) and was used to make spore suspensions. R2YE (Hopwood et al., 1985) was used for regenerating protoplasts and, after addition of the appropriate antibiotic, for selecting recombinants. M512 was used for promoter-probing experiments with pIJ2587 (G. P. van Wezel, J. White \& M. J. Bibb, unpublished results). S. ramocissimus ATCC 27529 was obtained from the ATCC culture collection, Manassas, VA, USA. E. coli JM109 (Messing et al., 1981) was the host for pUC18 (Yanisch-Perron et al., 1985) and constructs derived from it.

Liquid cultures were grown in TSBS medium [3\% (w/v) Difco tryptone soy broth with $10 \%(\mathrm{w} / \mathrm{v})$ sucrose], in yeast extract/malt extract medium (YEME) supplemented with $0.5 \%$ (w/v) glycine (Hopwood et al., 1985), or in minimal medium (MM) with $1 \%(\mathrm{w} / \mathrm{v})$ glucose as carbon source, and were inoculated at a density of approximately $5 \times 10^{6}$ c.f.u. $\mathrm{ml}^{-1}$ and grown at $30^{\circ} \mathrm{C}$ with vigorous shaking $(300$ r.p.m. $\left.\min ^{-1}\right)$. Cell growth was measured as increase in optical density at $600 \mathrm{~nm}$.

PCR conditions. PCRs were performed in a minicycler (MJ Research), using $P f u$ polymerase (Stratagene) and the buffer provided by the supplier, in the presence of $5 \%(\mathrm{v} / \mathrm{v})$ DMSO and $200 \mu \mathrm{M}$ dNTP. No additional $\mathrm{Mg}^{2+}$ was added to the reaction mixture. The following PCR programme was used: 30 cycles of $45 \mathrm{~s}$ melting at $94^{\circ} \mathrm{C}, 1 \mathrm{~min}$ annealing at $54^{\circ} \mathrm{C}$ and $90 \mathrm{~s}$ extension at $72^{\circ} \mathrm{C}$, followed by an additional $10 \mathrm{~min}$ at $72{ }^{\circ} \mathrm{C}$.

DNA manipulation and sequencing. DNA cloning, isolation and gel electrophoresis were performed by standard procedures (Sambrook et al., 1989). DNA was sequenced using the T7 DNA polymerase sequencing kit (Pharmacia). For sequencing of $r p s B$ and $t s f$, we used subclones in pUC18 as templates, and where necessary oligonucleotides were designed and used to fill in the gaps.

Southern hybridization. Genomic DNAs used for Southern analysis were isolated as described by Hopwood et al. (1985). For high-resolution hybridization experiments to investigate the presence of tsf in $S$. coelicolor and S. ramocissimus, 
(a)
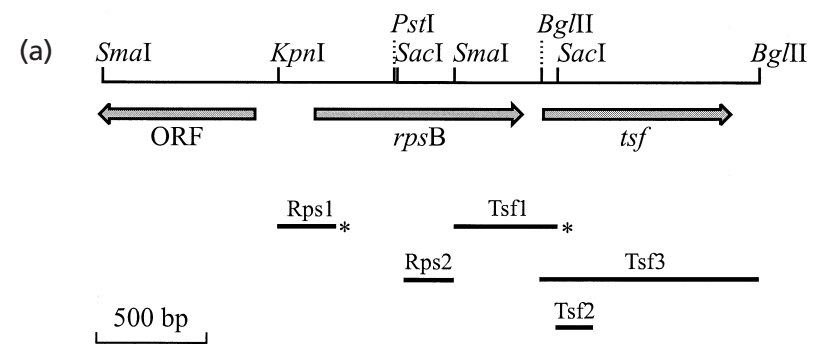

(b)

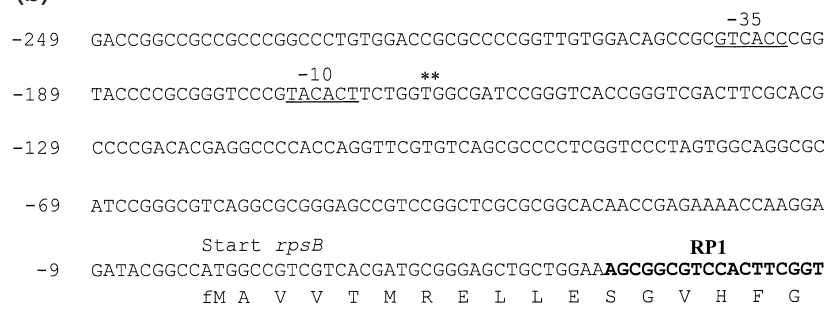

(c) GGCGAGAAGGCCGAGAAGAAGGACGACGCCGAGGCCGCCGAGAAGCCCGCCGAGGCCCCC

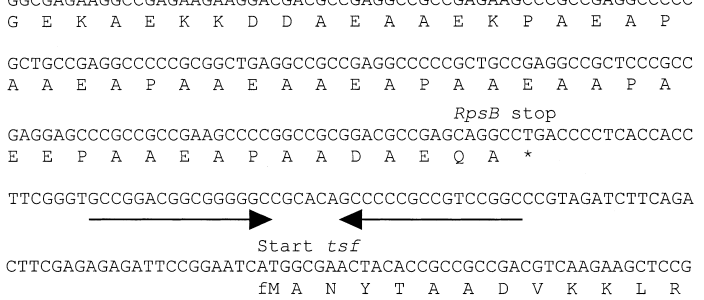

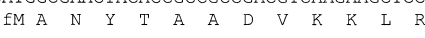

\begin{abstract}
Fig. 1. Map of the rpsB-tsf operon in Streptomyces coelicolor and sequences of the starts of the genes and upstream regions. (a) Restriction map of the rpsB-tsf operon. Filled arrows indicate the genes with their direction of transcription. Probes described in the Methods section are shown as solid lines under the map. Rps1 and Tsf1 are probes used for nuclease S1 mapping, with asterisks referring to ${ }^{32} \mathrm{P}$-labelled $5^{\prime}$ ends. Rps2, Tsf2 and Tsf3 were used for hybridization experiments. ORF encodes a putative regulatory protein of unknown function. (b) Nucleotide sequence of the start of $\operatorname{rps} B$ and upstream region. The deduced amino acid sequence of the ribosomal protein S2 is shown below the sequence. Nucleotides probably constituting the transcription start site are indicated by asterisks above the sequence; putative -35 and -10 regions are underlined. The sequence of oligonucleotide RP1, used for $P C R$ and for sequencing, is shown in bold. (c) Nucleotide sequence of the intergenic region between $\operatorname{rps} B$ and tsf. Deduced amino acid sequences such as for the A, E and P-rich C-terminal extension of $\mathrm{S} 2$ are shown below the sequence. Arrows show the $16 \mathrm{bp}$ inverted repeat possibly involved in attenuation of transcription by termination.
\end{abstract}

genomic DNA was digested with the appropriate enzymes and separated electrophoretically on a $0.7 \%$ agarose gel in TAE buffer, using the Gibco-BRL $1 \mathrm{~kb}$ ladder as DNA size markers. Agarose gels were pretreated and subsequently blotted on Hybond-N + nylon membranes (Amersham) using $20 \times$ SSC as the transfer buffer, basically according to Sambrook et al. (1989). Hybridization and washing conditions were as described previously (van Wezel et al., 1991).

Probes. As a probe for screening the M145 cosmid library we used Tsf2 (Fig. 1a), a fragment generated by PCR of $S$. coelicolor chromosomal DNA using oligonucleotides TSF1 and TSF2. The latter are multiply degenerated oligonucleotides designed to match highly conserved regions from known tsf genes and correspond to the sequences encoding amino acid residues $20-27$ and $75-82$ of E. coli EF-Ts, respectively. For Southern hybridization experiments we used Tsf3, a $1 \mathrm{~kb}$ BglII fragment from pUSCTs-1 harbouring the entire $S$. coelicolor tsf gene (Fig. 1a). Both probes were ${ }^{32} \mathrm{P}$ labelled by random priming (Feinberg \& Vogelstein, 1983).

Probe Rps1, used for mapping $r p s B-t s f$ transcripts, was generated by PCR amplification of a $300 \mathrm{bp}$ fragment with pUSCS2, a pUC18 derivative containing the KpnI/PstI DNA fragment harbouring the start of $r p s B$ and 191 bp of upstream region as template, and as primers the $\mathrm{pUC}$ reverse sequencing primer (16-mer) and oligonucleotide RP1 (Fig. 1b), the latter ${ }^{32} \mathrm{P}$-end-labelled at the $5^{\prime}$ end. Probe Tsf1 consisted of the 460 bp SmaI-SacI fragment encompassing the $r p s B-t s f$ intergenic region, uniquely ${ }^{32} \mathrm{P}$-end-labelled at the $\mathrm{SacI}$ site. For mapping rpsL-tuf1 transcripts, we used the tuf1 probe corresponding to the $200 \mathrm{bp}$ region encompassing the first $5 \mathrm{nt}$ of tuf1 and $195 \mathrm{nt}$ of upstream region (van Wezel et al., 1994). The probes used for nuclease S1 mapping harboured a significantly large non-homologous extension, typically around $50 \mathrm{bp}$ of vector sequence, to allow discrimination between DNA-RNA hybrids and reannealed probe. Finally, for Northern hybridization experiments we used Rps2, a $260 \mathrm{bp} \mathrm{SacI-SmaI} \mathrm{fragment} \mathrm{internal} \mathrm{to} r p s B$ (Fig. 1a), ${ }^{32} \mathrm{P}-$ labelled by random priming (Feinberg \& Vogelstein, 1983).

Promoter-probe experiments. The promoterless $S$. coelicolor redD gene present in pIJ2587 (G. P. van Wezel, J. White \& M. $\mathrm{J}$. Bibb, unpublished results) was used as a reporter gene for screening of in vivo promoter activity. Insertion of the $1.3 \mathrm{~kb}$ SmaI-Pst I fragment, containing the start of $r p s B$ and upstream sequences (Fig. 1a), into pIJ2587 resulted in pIJ2587-rpsB. To screen the $r p s B-t s f$ intergenic region for possible promoter activity, we cloned the $0.73 \mathrm{~kb}$ SacI fragment into pIJ2587, with the start of $t s f$ oriented towards the start of redD, resulting in pIJ2587-tsf. M512 transformants containing pIJ2587, pIJ2587-rpsB or pIJ2587-tsf were grown on R2YE and $\mathrm{MM}$ in the presence of $10 \mu \mathrm{g}$ thiostrepton $\mathrm{ml}^{-1}$. Undecylprodigiosin production was assessed visually.

RNA isolation. RNA was isolated from M145 according to Hopwood et al. (1985). To remove residual DNA, the RNA was salt-precipitated in $3 \mathrm{M}$ sodium acetate $(\mathrm{pH} \mathrm{6.0)}$ and subsequently treated with DNase I $\left(37^{\circ} \mathrm{C}\right.$ for $1 \mathrm{~h}$, with $0 \cdot 1 \mathrm{U}$ DNase I per $50 \mathrm{ml}$ initial culture sample). Samples were then extracted with a 1:1 mixture of phenol and chloroform (saturated with $100 \mathrm{mM}$ Tris $\mathrm{pH} 7 \cdot 0$ ) and precipitated in $0.4 \mathrm{M}$ sodium acetate ( $\mathrm{pH} \mathrm{6.0)}$ with 2-propanol. The RNA was resuspended in water and the concentration determined spectrophotometrically.

Nuclease S1 mapping. RNA (10 $\mu$ g) was hybridized with the appropriate DNA probe according to Murray (1986) in TCA buffer (Summerton et al., 1983). All subsequent steps were carried out as described previously (Strauch et al., 1991), using an excess of probe.

Northern analysis. RNA samples (approx. $10 \mu \mathrm{g}$ ) were glyoxylated, run in a $1.2 \%$ agarose gel in $20 \mathrm{mM}$ sodium phosphate buffer ( $\mathrm{pH} \mathrm{6.7)}$ and transferred to Hybond-N+ nylon membranes using $30 \mathrm{mM}$ sodium phosphate $(\mathrm{pH} 6.7)$ as the blotting buffer. Hybridization and washing conditions were as described previously (Tieleman et al., 1997).

Computer analysis. The BLAST search engines BLASTN, BLASTP and BLASTX (Altschul et al., 1997) were used to perform 
database searches; the Wisconsin GCG Package (Devereux et al., 1984) for sequence alignments, protein analysis and prediction of RNA secondary structures; and RASMOL (Sayle \& Milner-White, 1995) for drawing the EF-Tu-EF-Ts structure.

\section{RESULTS}

\section{S. coelicolor and S. ramocissimus have one tsf gene}

The multiplicity and divergence of $t u f$ genes in various Streptomyces species, including S. coelicolor (two tuf genes) and $S$. ramocissimus (three tuf genes), prompted investigation into the number of $t s f$ genes in these organisms, and comparison of the predicted structure of the respective gene products to that of other known EFTs proteins.

An internal fragment of the tsf gene from $S$. coelicolor, designated Tsf2, was obtained by PCR, using S. coelicolor A3(2) M145 total DNA as template and as primers oligonucleotides TSF1 and TSF2 (see Methods section and Fig. 1a). Tsf2 was hybridized to the cosmid library of S. coelicolor M145 (Redenbach et al., 1996), allowing identification of a cosmid clone harbouring the complete tsf gene and flanking regions. tsf was identified on the overlapping cosmids 2E1 (GenBank accession no. AL023797) and 6A9 (AL031035), located between 5 and 6 o'clock on the chromosomal map. A $2 \cdot 1 \mathrm{~kb} K p n \mathrm{I}-\mathrm{Bg}$ lII fragment was isolated from cosmid 2E1 and subcloned into KpnI/BamHI-digested pUC18, resulting in pUSCTs-1. A restriction map is shown in Fig. 1(a).

To check whether more than one tsf gene is present in $S$. coelicolor or $S$. ramocissimus, genomic DNA was isolated from each of these strains, digested with $\mathrm{BamHI}$ or Pst I and fractionated by agarose gel electrophoresis. The gel was blotted and hybridized with Tsf3 (Fig. 1a) at low stringency to identify all genes with some similarity to tsf. A single hybridizing band was observed in each lane, indicating that only one tsf gene occurs in both organisms. A representive result is shown in Fig. 2.

\section{Sequence analysis of the $S$. coelicolor rpsB-tsf operon and of S. ramocissimus tsf}

Sequence analysis of the insert of pUSCTs- 1 revealed two ORFs (GenBank accession no. AF034101), the upstream ORF strongly resembling E. coli rpsB (encoding ribosomal protein S2) and the downstream ORF with high homology to E. coli tsf (encoding EF-Ts). This gene organization is typical of eubacteria (An et al., 1981; Lindahl \& Zengel, 1986).

Sequence analysis of the chromosomal region around the $r p s B-t s f$ operon in the $S$. coelicolor A3(2) genome has recently been completed (Sanger Centre, Cambridge, UK; sequencing data can be obtained from ftp://ftp. sanger.ac.uk/pub/S_coelicolor/sequences). The data indicate that the $r p s B-t s f$ gene cluster is located downstream of a putative regulatory gene, which in turn is preceded by the developmental sigma factor gene whiG

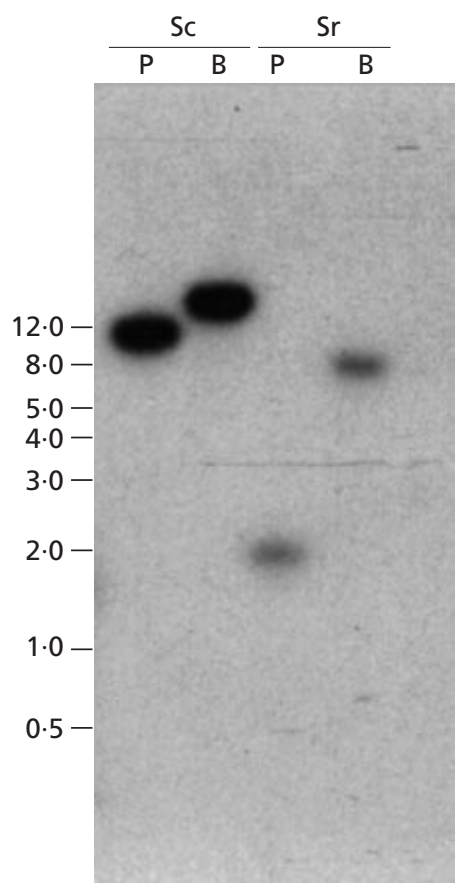

Fig. 2. Southern hybridization of $S$. coelicolor $A 3(2)$ and $S$. ramocissimus total DNA samples. Sc, $S$. coelicolor DNA; Sr, $S$. ramocissimus DNA; $\mathrm{P}$, Pstl digest; $\mathrm{B}, \mathrm{BamHI}$ digest. DNA size markers (in kb) are shown on the left.

(Chater et al., 1989). Downstream of $r p s B-t s f$ lies $p y r H$, a gene involved in pyrimidine biosynthesis.

The S. coelicolor S2 protein consists of 310 aa with sequence identity to approximately $50 \%$ of the 241 aa in E. coli S2 (GenBank accession no. P02351) and carries a C-terminal domain significantly larger than that of most other S2 homologues in eubacteria, with an extension consisting of a repetitive amino acid sequence (Fig. 1b). Considering the unusual nature of the C-terminal extension, and the presence of an out-of-frame stop codon immediately in front of it, the sequence of this region is of particular interest. Its accuracy has therefore been confirmed by extensive DNA sequencing carried out by the Sanger Centre on both cosmids 2E1 and 6A9. The only other example of an S2 protein with a repetitive sequence in its C-terminal region was found in $\mathrm{Myco-}$ bacterium tuberculosis (GenBank accession no. Q10796), but the extension shares no significant homology to that of $S$. coelicolor EF-Ts.

\section{Comparison of the $S$. coelicolor and S. ramocissimus tsf genes with $E$. coli tsf}

The $S$. ramocissimus tsf gene was obtained by PCR on chromosomal DNA using oligonucleotides based on the sequence of the $S$. coelicolor $r p s B-t s f$ gene cluster. A $1.45 \mathrm{~kb}$ DNA fragment containing the $S$. ramocissimus tsf gene and upstream sequences was amplified using oligonucleotides corresponding to nt positions 


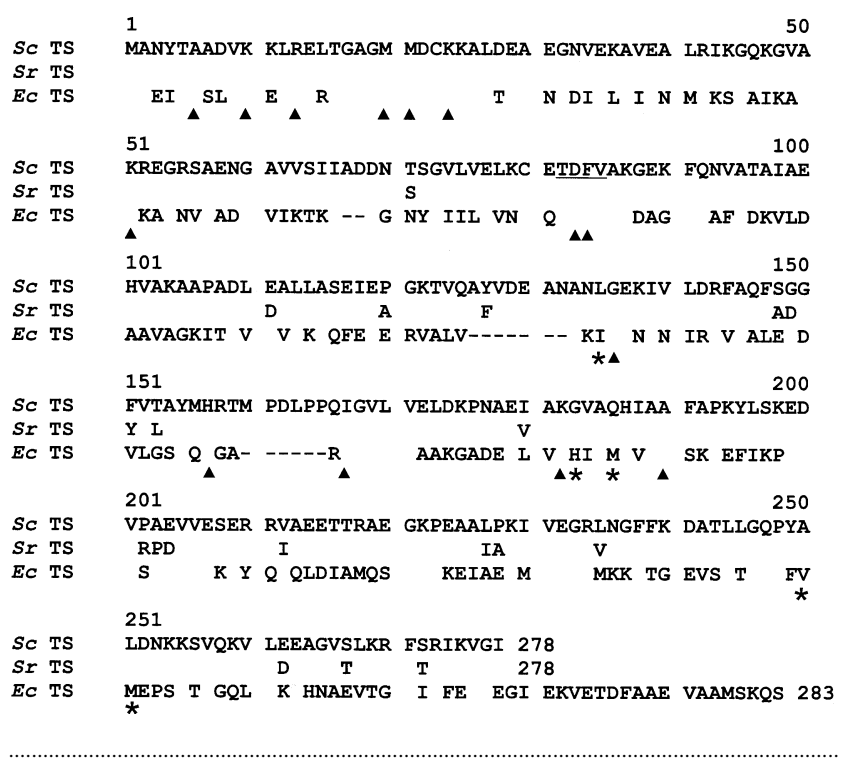

Fig. 3. Amino acid comparison between EF-Ts homologues of $S$. coelicolor, S. ramocissimus and E. coli. Numbers refer to the $S$. coelicolor EF-Ts amino acid sequence. For $S$. ramocissimus and $E$. coli EF-Ts, only differences with $\mathrm{S}$. coelicolor EF-Ts are shown. SC TS, S. coelicolor EF-Ts; Sr TS, S. ramocissimus EF-Ts; Ec TS, E. coli EF-Ts. The underlined letters TDFV indicate the conserved EF-Ts signature. Triangles and asterisks refer to residues shown for $E$. coli EF-Ts to be in direct contact with EF-Tu; triangles represent contact residues conserved among the three EF-Ts species, and asterisks show residues that are different between $E$. coli and Streptomyces EF-Ts.

$-529 /-510$ and $+939 /+959$ with respect to the start of $S$. coelicolor tsf, respectively. The $0.95 \mathrm{~kb} B g l \mathrm{II}$ segment internal to the PCR-amplified DNA fragment was inserted into BamHI-digested pUC18, resulting in pUSRTs-1, and the DNA sequence of the insert was determined (GenBank accession no. AF130345).

The $S$. coelicolor and $S$. ramocissimus tsf genes display high sequence homology and encode a protein of 278 aa. A sequence alignment of the derived EF-Ts gene products of E. coli, S. coelicolor and S. ramocissimus tsf is shown in Fig. 3. The Streptomyces gene products show $93 \%$ identical amino acids, and exhibit approximately $40 \%$ amino acid sequence identity with E. coli EF-Ts. The N-terminal parts of the proteins share significantly higher similarity than the middle and Cterminal parts. The EF-Ts signature TDFV (underlined in Fig. 3) is conserved among the three EF-Ts species. Residues reported to be involved in interaction with EF-Tu in the crystallized EF-Tu.EF-Ts complex (Kawashima et al., 1996) are shown under the alignment. Triangles represent contact residues conserved among all three EF-Ts species, while asterisks represent contact residues that differ between E. coli and Streptomyces EFTs (Fig. 3). Interestingly, while all contact residues conserved in EF-Ts of E. coli and Streptomyces (triangles) correspond to residues in EF-Tu that are conserved in the three different EF-Tu species (EF-Tu1, 2 and 3), those contact residues that are different in the Streptomyces EF-Ts (asterisks) correspond to putative contact residues in EF-Tu1 and EF-Tu3 that are also different.

\section{In vivo promoter probing of the S. coelicolor rpsB-tsf gene cluster}

To determine the presence and approximate location of promoter(s) of the rpsB-tsf gene cluster, we used the promoter-probe vector pIJ2587 (G. P. van Wezel, J. White \& M. J. Bibb, unpublished results), which contains the promoter-less redD gene, encoding the transcriptional activator of biosynthetic genes for the antibiotic undecylprodigiosin, also called Red (Narva \& Feitelson, 1990). Introduction of pIJ2587 containing promoter sequences upstream of redD into $S$. coelicolor M512 (M145 $\Delta r e d D, \Delta a c t I I-O R F 4)$ resulted in production of RedD, and hence activation of the Red cluster.

The upstream region of $S$. coelicolor $r p s B$ was cloned as a $1.3 \mathrm{~kb}$ SmaI-PstI fragment into pIJ2587 digested with the same enzymes, and the rpsB-tsf intergenic region was cloned as a $0.73 \mathrm{~kb} \mathrm{SacI}$ fragment into SacI-digested pIJ2587 with the start of $t s f$ proximal to $r e d D$, resulting in $\mathrm{pIJ} 2587-r p s B$ and $\mathrm{pIJ} 2587-t s f$, respectively. Introduction of pIJ2587-rpsB resulted in strong Red production as soon as recombinant colonies could be discerned, indicative of the presence of at least one promoter in the SmaI-PstI fragment harbouring the start of $r p s B$ and $980 \mathrm{bp}$ of upstream region. The lack of any trace of Red production by recombinant M512 containing pIJ2587-tsf was similar to the result with control transformants harbouring pIJ2587 without an insert, and suggested that transcription is not initiated in the $r p s B-t s f$ intergenic region.

\section{The rpsB-tsf gene cluster is transcribed from a single promoter}

To establish the level and timing of $r p s B$ transcription in vivo, RNA was isolated from mycelium harvested from $S$. coelicolor cultures grown in MM with $1 \%$ glucose. RNA samples were analysed by high-resolution nuclease S1 mapping, using Rps1 (Fig. 1a) as the probe, and the protected fragment was analysed alongside a DNA sequence ladder produced with oligonucleotide RP1 as sequencing primer. A representative result is shown in Fig. 4. A band corresponding to $r p s B$ transcripts could be readily identified in RNA isolated at all time points; the signals arising from RNA isolated during early exponential phase (lanes $1-2$ ) were significantly more intense than those arising from RNA isolated in later growth phases (lanes 3-6). The rpsB transcript corresponds to a transcriptional start site 161-162 nt upstream of the $r p s B$ translational start site. The putative transcriptional start site is preceded by the sequences GTCACC around -35 and TACACT around -10 , which are separated by 19 bp (Fig. 1b). These sequences show low but relevant similarity to the consensus sequence for the major class of eubacterial promoters, TTGACA around -35 and TATAAT around -10 


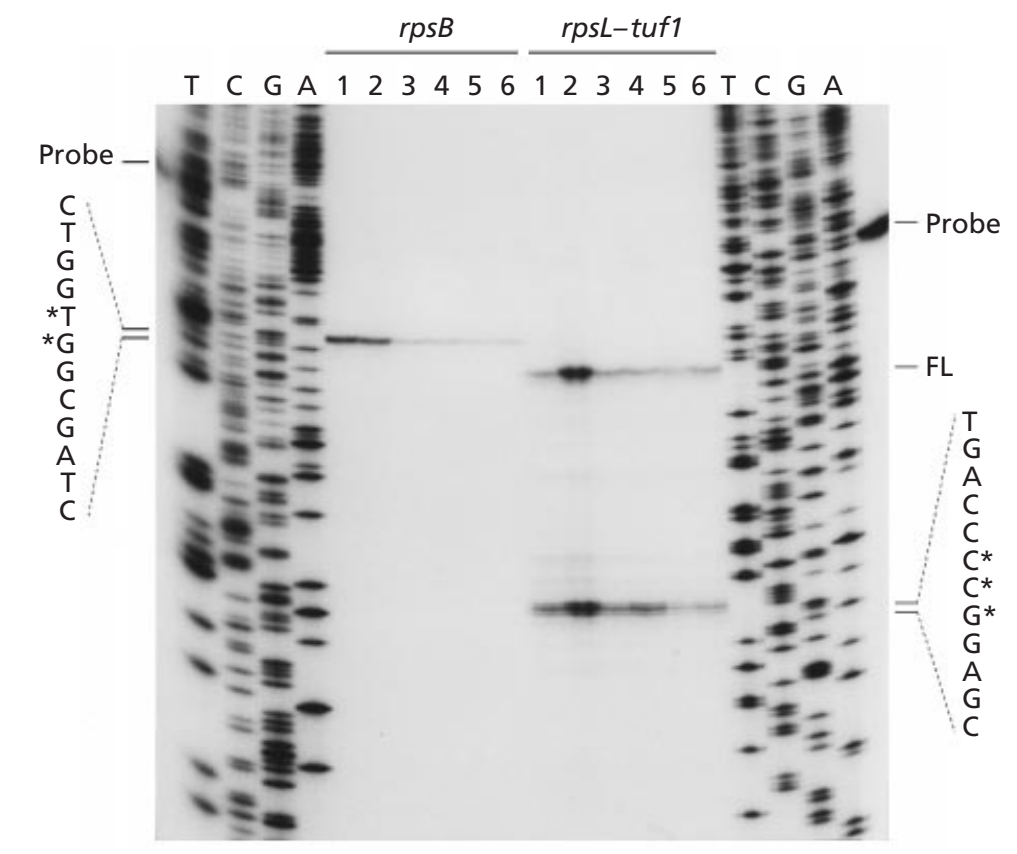

Fig. 4. Transcription of $r p s B-t s f$ and tuf1 in MM-grown cultures of $S$. coelicolor. Lanes T, $C, G$ and A, DNA sequence ladder; asterisks refer to most probable transcription start sites. Probe, full-length DNA probes used for nuclease S1 mapping (including nonhomologous 3' extension). FL, full-length protection. RNA samples were applied from cells harvested at the following $\mathrm{OD}_{600}$ values: lanes 1 and $2,0.2$ and 0.4 , respectively (early exponential phase); lanes 3 and $4,0.7$ and 1.0 , respectively (midexponential phase); lane 5, 1.3 (transition phase); lane 6, 1.5 (stationary phase).

(Hawley \& McClure, 1983). In S. coelicolor, such canonical sequences appear to be recognized by $\sigma^{\text {hrdB }}$ (Brown et al., 1992).

To confirm the data obtained by promoter probing, we used Tsf1, harbouring the rpsB-tsf intergenic region (Fig. 1a), as a probe for nuclease S1 mapping of the same RNA samples used for mapping $r p s B$ transcripts. As expected, we detected only a signal corresponding to full-length protection of the probe, indicating that no promoter is located immediately upstream of $t s f$, and that $r p s B$ and $t s f$ are transcribed from a single promoter upstream of $r p s B$ (data not shown).

We also analysed tuf1 transcription in S. coelicolor by nuclease S1 mapping, using the $200 \mathrm{bp}$ tuf1 probe. Comparison of rpsB-tsf and tuf1 transcription (Fig. 4) shows that timing of expression is similar, although $r p s B$ transcripts reach a maximum somewhat earlier than tuf1. Two tuf1 transcripts were identified, one originating from a promoter with a transcription start site $125 \mathrm{nt}$ upstream of the start of the gene (designated tuf $1 \mathrm{p})$, and one corresponding to full-length protection of the probe, indicative of a distal promoter, and most likely the $r p s L$ operon promoter ( $r p s L \mathrm{p}$ ). While both promoters show the same growth-phase-dependence, tuf1p apparently contributes more to tuf1 transcription under these conditions than rpsLp. This regulation is similar to that observed for the $S$. ramocissimus rpsL operon (Tieleman et al., 1997). Transcription probably starts at the $G$ residue at position -125 relative to the start of tuf1, which is 2 nt downstream of the transcription start site predicted by Tieleman et al. (1997) on the basis of sequence homology to $S$. ramocissimus tuf1.

In E. coli, $r p s B$ is expressed more abundantly than $t s f$, resulting in a $3: 1$ ratio of the respective gene products, a

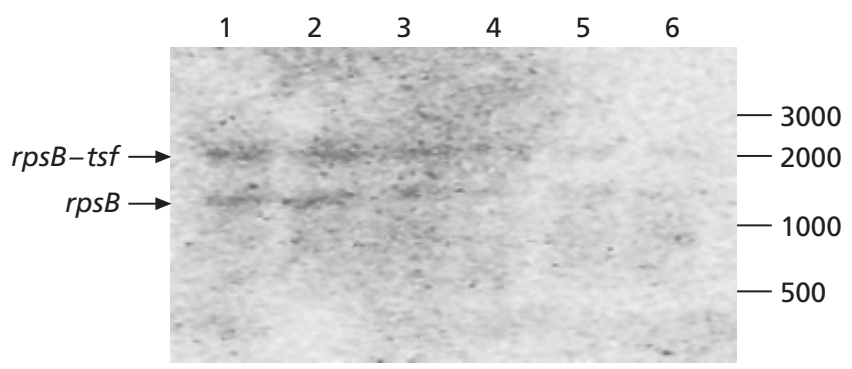

Fig. 5. Northern analysis of $r p s B-t s f$ transcription. RNA applied to lanes was the same as described for Fig. 4. $r p s B$, monocistronic $r p s B$ transcript; rpsB-tsf, bicistronic rpsB-tsf transcript. RNA size markers (in $n t$ ) are shown on the right.

phenomenon possibly due to terminator sequences immediately downstream of rpsB (An et al., 1981). To establish whether such a transcriptional regulation occurs in S. coelicolor, the same RNA as used for nuclease S1 mapping experiments was analysed by Northern blotting using Rps2 (Fig. 1a) as the probe. The experiment confirmed the growth-phase-dependent regulation of the $S$. coelicolor rpsB-tsf operon (Fig. 5). Interestingly, two bands could be detected in all lanes, corresponding to transcripts with sizes of approximately 1100 and $2100 \mathrm{nt}$. Since we have identified the exact location of the $r p s B$ promoter (Fig. 1b), we can conclude that the smaller band corresponds to the monocistronic $r p s B$ transcript, while the larger band corresponds to the bicistronic $r p s B-t s f$ transcript. The approximately equal intensity of the bands suggests a $2: 1$ molar ratio of $r p s B$ transcripts versus tsf transcripts. The apparent termination immediately downstream of rpsB may be 
explained by the presence in this region of a perfect inverted repeat, consisting of two elements of $16 \mathrm{bp}$, followed by an unusually A $+\mathrm{T}$-rich region (Fig. 1b).

\section{DISCUSSION}

Southern analysis of $S$. coelicolor and S. ramocissimus total DNA samples revealed one $t s f$ gene, corresponding to a single species of elongation factor EF-Ts that apparently interacts with different species of elongation factor EF-Tu in these strains. This suggests a surprising adaptability for EF-Ts as the guanine-nucleotide exchange factor, since EF-Tu1 and EF-Tu3 show only approximately $65 \%$ amino acid identity, while EF-Tu2, so far found only in S. ramocissimus, shows $88 \%$ identical residues with EF-Tu1 (van Wezel et al., 1994; Vijgenboom et al., 1994). The actual functioning as a real elongation factor of a protein as divergent as EF$\mathrm{Tu} 3$ was indeed proven in vitro in a poly $(\mathrm{U})$ translating system containing a complete $S$. coelicolor extract with EF-Tu3 in the absence of EF-Tu1 (L. N. OlsthoornTieleman, L. Plooster \& B. Kraal, unpublished results). Here, we have demonstrated that the EF-Ts in such an extract is derived from a single source, the tsf gene located in an operon with $r p s B$.

The regular EF-Tu1 is an abundant cytoplasmic protein occurring at a considerable molar excess over ribosomes. This implies that expression of the distal tuf1 in the rpsL operon in Streptomyces is individually regulated, since the other three genes express ribosomal proteins $\mathrm{S} 12$ and $S 7$ and elongation factor $\mathrm{G}$ at levels about equimolar to that of ribosomes. Indeed, we showed that tuf1 is transcribed not only from the rpsL promoter for full operon transcription, but also from a second promoter located in the intergenic region between fus and tuf1 of S. ramocissimus (Tieleman et al., 1997) and S. coelicolor (Fig. 4). In the case of S. coelicolor, tuf1p contributes at least as much to transcript levels during normal growth as rpsLp. For the rpsB operon, the opposite situation prevails since transcription of the distal tsf is expected to be significantly weaker than that of $r p s B$. In our in vivo promoter probing assays, the $\operatorname{Red}^{+}$phenotype of $S$. coelicolor M512 transformants containing pIJ2587-rpsB revealed a promoter within $980 \mathrm{nt}$ upstream of the $r p s B$ translational start site, and the failure of transformants containing pIJ2587-tsf to synthesize Red indicated that promoter activity was absent from the rpsB-tsf intergenic region.

Transcription analysis by nuclease S1 mapping of the $r p s B$ start region detected $r p s B$ operon transcripts in $S$. coelicolor RNA isolated from all growth phases, with the highest transcript levels during early exponential growth (Fig. 4). This suggests that $r p s B-t s f$ transcription shows growth-phase-dependent regulation typical of genes for translational components (Lindahl \& Zengel, 1986). Similar analysis of tuf1 transcription (the latter located in the rspL operon) showed that both operons are transcribed in a similar way, although $r p s B$ operon transcripts reached peak levels earlier (at an $\mathrm{OD}_{600}$ below 0.2) than tuf1 transcripts $\left(\mathrm{OD}_{600} 0 \cdot 4\right)$.
The putative -35 and -10 sequences of the $r p s B-t s f$ promoter (GTCACC and TACACT, separated by $19 \mathrm{bp}$ ) show 3 and 4 out of $6 \mathrm{nt}$ matches, respectively, with consensus sequences for the major class of eubacterial promoters (TTGACA and TATAAT, separated by 16-18 bp; Hawley \& McClure, 1983). Many Streptomyces promoters show relatively low similarity to the consensus promoter (Strohl, 1992), especially around the -35 sequence, and the timing of expression of the $r p s B-t s f$ operon suggests that its regulation is similar to other operons for proteins involved in translation.

Further transcription analysis by nuclease S1 mapping of the $r p s B-t s f$ intergenic region revealed only a single band corresponding to read-through from an upstream promoter (data not shown). As in E. coli (Bendiak \& Friesen, 1981), this indicates that there is no promoter immediately upstream of $t s f$, and that $r p s B$ and $t s f$ are transcribed from a single promoter upstream of $r p s B$.

An obvious way of regulating transcription and enhancing expression of $r p s B$ in comparison to $t s f$ could be found in the presence of an additional terminator in the $r p s B-t s f$ intergenic region. Indeed, Northern blotting of the S. coelicolor M145 RNA used for nuclease S1 mapping with the Rps2 probe revealed two bands with sizes corresponding to the full operon length and an additional $r p s B$ transcript (Fig. 5). Such an attenuation mechanism had already been postulated by An et al. (1981) for the rpsB-tsf operon in E. coli on the basis of a long but imperfect inverted repeat in the intergenic region. In the intergenic region of the $S$. coelicolor $r p s B-t s f$ operon we see a perfect inverted repeat consisting of two $16 \mathrm{bp}$ elements, possibly allowing Rhoindependent termination of the RNA polymerase. A different attenuation mechanism was proposed for the rpsB-tsf operon of Spiroplasma citri, where a DNAbinding protein was shown to interact with the region immediately downstream of $r p s B$ (Le Dantec et al., $1998 \mathrm{a}, \mathrm{b})$. Processing of the $r p s B-t s f$ transcript at the inverted repeat is unlikely since S1 nuclease mapping by means of the Tsf1 probe gave only one signal corresponding to full-length protection.

While most eubacterial S2 proteins contain approximately 240 aa residues, for example 241 aa in E. coli (GenBank accession no. P02351), 246 aa in Bacillus subtilis (P21464) and 251 aa in Haemophilus influenzae (P44371), sequence comparison showed S. coelicolor S2 to be a 310 a protein with a 70 aa C-terminal extension. The highly repetitive 46 aa in the C-terminal region of $S$. coelicolor S2 consist almost exclusively of Ala, Glu and Pro residues. The S2 homologue of the related actinomycete M. tuberculosis (286 aa; Q10796) also has a repetitive sequence, but it shows no relevant similarity to the extension of S. coelicolor S2. The function of the repetitive $\mathrm{C}$-terminal extension, which may be limited to members of the actinomycete family, is unknown.

The so-called EF-Ts signature sequence TDFV is strictly conserved and is essential for interaction with EF-Tu. From the crystal structure of the E. coli EF-Tu.EF-Ts 


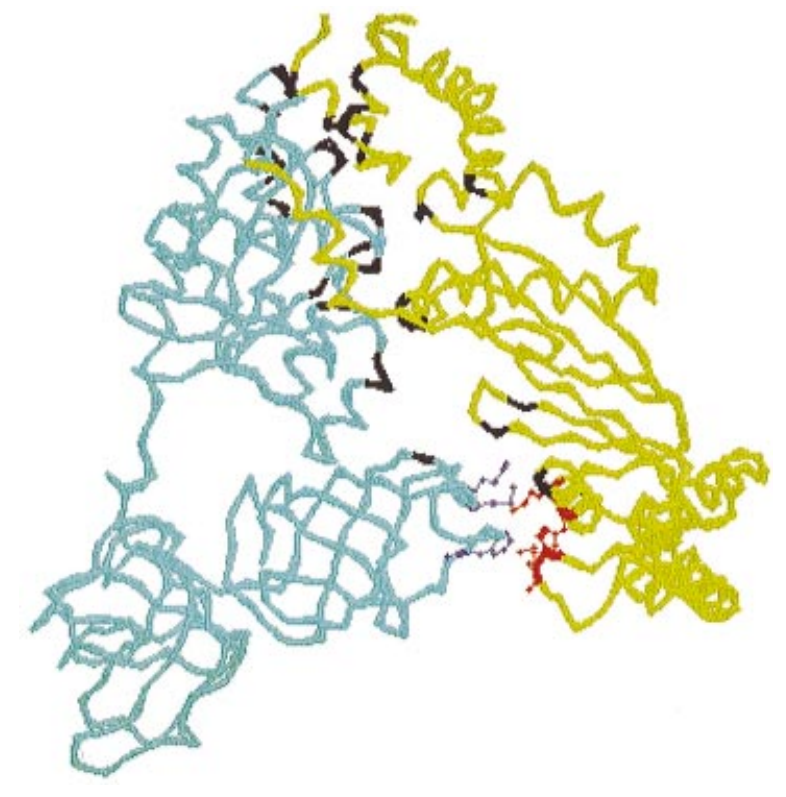

Fig. 6. Three-dimensional structure of the EF-Tu.EF-Ts complex of E. coli, based on the results of Kawashima et al. (1996). The EF-Tu moiety is represented in light blue, the EF-Ts moiety in yellow. Amino acids in other colours represent residues involved in the direct contact between EF-Tu and EF-Ts: black, conserved in E. coli, S. coelicolor and S. ramocissimus; purple, different between Streptomyces EF-Tu1 and EF-Tu3; red, different between $E$. coli and Streptomyces EF-Ts.

complex (Fig. 6), Kawashima et al. (1996) concluded that these residues are involved in dislocating the magnesium ion from EF-Tu, forcing GDP to dissociate from the molecule. Residues known to be involved in the direct interaction between EF-Tu (backbone represented in light blue) and EF-Ts (yellow) are highlighted in Fig. 6. Amino acids coloured black in the guanine-nucleotide-binding domain of the EF-Tu moiety represent interaction residues also conserved in Streptomyces EFTu1, 2 and 3, and the black ones in the EF-Ts moiety represent the contact residues also conserved in $S$. coelicolor and S. ramocissimus EF-Ts. Interestingly, when interaction residues in the EF-Tu moiety of the complex show divergence among the three Streptomyces species, i.e. aa 323, 349 and 351 in the E. coli numbering (indicated in purple), it transpires that the corresponding residues in EF-Ts, namely aa 167, 170, 234 and 235 in E. coli numbering (red) are also changed.

In a mutational analysis of the roles of E. coli EF-Ts residues in the interaction with EF-Tu, Zhang et al. (1998) concluded that this interaction is a global event in which multiple small conformational changes result in a significant cumulative rearrangement of the guaninenucleotide-binding domain of EF-Tu, promoting GDP release. In the interaction of Streptomyces EF-Ts with its divergent EF-Tu partners, it seems that covariation of normally conserved residues has led to a renewed balance for sufficient catalysis of the GDP release of all EF-Tu species. This is now open to experimental verification.

\section{ACKNOWLEDGEMENTS}

We thank Estíbaliz Sánchez Meroño (supported by a grant from the European Socrates Students Exchange Programme) for her experimental contribution. We are grateful to Helen Kieser (John Innes Centre, Norwich) for screening the $S$. coelicolor cosmid library and for providing cosmids $2 \mathrm{E} 1$ and 6A9, and to Julian Parkhill (Sanger Centre, Cambridge) for help with sequence annotation and for discussions.

We would like to dedicate this paper to Professor Sir David Hopwood on the occasion of his retirement.

\section{REFERENCES}

Altschul, S. F., Madden, T. L., Schaffer, A. A., Zhang, J., Zhang, Z., Miller, W. \& Lipman, D. (1997). Gapped BLAST and PSI-BLAST: a new generation of protein database search programs. Nucleic Acids Res 25, 3389-3402.

An, G., Bendiak, D. S., Mamelak, L. A. \& Friesen, J. D. (1981). Organization and nucleotide sequence of a new ribosomal operon in Escherichia coli containing the genes for ribosomal protein S2 and elongation factor Ts. Nucleic Acids Res 9, 4163-4172.

Bendiak, D. S. \& Friesen, J. D. (1981). Organization of genes in the four minute region of the Escherichia coli chromosome: evidence that $r p s B$ and $t s f$ are cotranscribed. Mol Gen Genet 181, 356-362.

Bourne, H. R., Sanders, D. A. \& McCormick, F. (1991). The GTPase superfamily: conserved structure and molecular mechanism. Nature 349, 117-127.

Brown, K. L., Wood, S. \& Buttner, M. J. (1992). Isolation and characterization of the major vegetative RNA polymerase of Streptomyces coelicolor A3(2); renaturation of a sigma subunit using GroEL. Mol Microbiol 6, 1133-1139.

Chater, K. F., Bruton, C. J., Plaskitt, K. A., Buttner, M. J., Méndez, C. \& Helmann, J. D. (1989). The developmental fate of $S$. coelicolor hyphae depends on a gene product homologous with the motility $\sigma$ factor of B. subtilis. Cell 59, 133-143.

van Delft, J. H. M., Verbeek, H. M., de Jong, P. J. \& Bosch, L. (1987). Transcription of the tRNA-tufB operon of Escherichia coli: activation, termination and antitermination. Nucleic Acids Res 15, 9515-9530.

Devereux, J., Haeberli, P. \& Smithies, O. (1984). A comprehensive set of sequence analysis programs for the VAX. Nucleic Acids Res 12, 387-395.

Feinberg, A. P. \& Vogelstein, B. (1983). A technique for radiolabelling of DNA restriction endonuclease fragments to high specific activity. Anal Biochem 132, 6-13.

Floriano, B. \& Bibb, M. J. (1996). $A f_{s} R$ is a pleiotropic but conditionally required regulatory gene for antibiotic production in Streptomyces coelicolor A3(2). Mol Microbiol 21, 385-396.

Hawley, D. K. \& McClure, W. R. (1983). Compilation and analysis of Escherichia coli promoter DNA sequences. Nucleic Acids Res 11, 2237-2255.

Hobbs, G., Frazer, C. M., Gardner, D. C. J., Flett, F. \& Oliver, S. G. (1989). Dispersed growth of Streptomyces in liquid culture. Appl Microbiol Biotechnol 31, 272-277.

Hopwood, D. A., Bibb, M. J., Chater, K. F. \& 7 other authors (1985). Genetic Manipulation of Streptomyces: a Laboratory Manual. Norwich: John Innes Foundation.

Hopwood, D. A., Chater, K. F. \& Bibb, M. J. (1995). Genetics of antibiotic production in Streptomyces coelicolor A3(2), a model streptomycete. Biotechnology 28, 65-102.

Jinks-Robertson, S. \& Nomura, M. (1987). Ribosomes and tRNA. In Escherichia coli and Salmonella typhimurium: Cellular and 
Molecular Biology, vol. 2, pp. 1358-1385. Edited by F. C. Neidhardt and others. Washington, DC: American Society for Microbiology.

Kawashima, T., Berthet-Colominas, C., Wulff, M., Cusack, S. \& Leberman, R. (1996). The structure of the Escherichia coli EF$\mathrm{Tu}$.EF-Ts complex at $2 \cdot 5 \AA$ resolution. Nature 379, 511-518.

Kraal, B., Bosch, L., Mesters, J. R., de Graaf, J. M., Woudt, L. P., Vijgenboom, E., Heinstra, P. W. H., Zeef, L. A. H. \& Boon, C. (1993). Elongation factors in protein synthesis. In The GTPase Superfamily, pp. 28-52. Edited by J. Marsh \& J. Goode. Chichester: Wiley.

Krab, I. M. \& Parmeggiani, A. (1998). EF-Tu, a GTPase odyssey. Biochim Biophys Acta 1443, 1-22.

Le Dantec, L., Bové, J. M. \& Saillard, C. (1998a). Gene organization and transcriptional analysis of the Spiroplasma citri $r p s B / t s f / x$ operon. Curr Microbiol 37, 269-273.

Le Dantec, L., Castroviejo, M., Bové, J. M. \& Saillard, C. (1998b). Purification, cloning, and preliminary characterization of a Spiroplasma citri ribosomal protein with DNA binding capacity. J Biol Chem 273, 24379-24386.

Lee, J. S., An, G., Friesen, J. D. \& Fiil, N. P. (1981). Location of the tufB promoter of Escherichia coli: cotranscription of $t u f B$ with four transfer RNA genes. Cell 25, 251-258.

Lindahl, L. \& Zengel, J. M. (1986). Ribosomal protein genes in Escherichia coli. Annu Rev Genet 20, 297-326.

van der Meide, P., Vijgenboom, E., Dicke, M. \& Bosch, L. (1982). Regulation of the expression of $t u f A$ and $t u f B$, the two genes encoding the elongation factor EF-Tu in Escherichia coli. FEBS Lett 139, 325-330.

Messing, J., Crea, R. \& Seeburg, P. H. (1981). A system for shotgun DNA sequencing. Nucleic Acids Res 9, 309-321.

Miyajima, A., Shibuya, M., Kuchino, Y. \& Kaziro, Y. (1981). Transcription of the E. coli tufB gene: cotranscription with four tRNA genes and inhibition by guanosine- $5^{\prime}$-diphosphate-3'diphosphate. Mol Gen Genet 183, 13-19.

Murray, M. G. (1986). Use of sodium trichloroacetate and mung bean nuclease to increase sensitivity and precision during transcript mapping. Anal Biochem 158, 165-170.

Narva, K. E. \& Feitelson, J. S. (1990). Nucleotide sequence and transcriptional analysis of the redD locus of Streptomyces coelicolor A3(2). J Bacteriol 172, 326-333.

Redenbach, M., Kieser, H. M., Denapaite, D., Eichner, A., Cullum, J., Kinashi, H. \& Hopwood, D. A. (1996). A set of ordered cosmids and a detailed genetic and physical map for the $8 \mathrm{Mb}$ Streptomyces coelicolor A3(2) chromosome. Mol Microbiol 21, 77-96.
Sambrook, J., Fritsch, E. F. \& Maniatis, T. (1989). Molecular Cloning: a Laboratory Manual, 2nd edn. Cold Spring Harbor, NY : Cold Spring Harbor Laboratory.

Sayle, R. A., \& Milner-White, E. J. (1995) RASMOL: biomolecular graphics for all. Trends Biochem Sci 20, 374.

Strauch, E., Takano, E., Baylis, H. A. \& Bibb, M. J. (1991). The stringent response in Streptomyces coelicolor A3(2). Mol Microbiol 5, 289-298.

Strohl, W. R. (1992). Compilation and analysis of DNA sequences associated with apparent streptomycete promoters. Nucleic Acids Res 20, 961-974.

Summerton, J., Atkins, T. \& Bestwick, R. (1983). A rapid method for preparation of bacterial plasmids. Anal Biochem 133, 79-84.

Tieleman, L. N., van Wezel, G. P., Bibb, M. J. \& Kraal, B. (1997). Growth phase-dependent transcription of the Streptomyces ramocissimus tuf1 gene occurs from two promoters. J Bacteriol 179, 3619-3624.

Vijgenboom, E., Woudt, L. P., Heinstra, P. W. H., Rietveld, K., van Haarlem, J., van Wezel, G. P., Shochat, S. \& Bosch, L. (1994). Three tuf-like genes in the kirromycin producer Streptomyces ramocissimus. Microbiology 140, 983-998.

van Wezel, G. P. (1994). Transcriptional regulation of translational genes in Streptomyces coelicolor A3(2). PhD thesis, Leiden University, The Netherlands.

van Wezel, G. P., Vijgenboom, E. \& Bosch, L. (1991). A comparative study of the rRNA operons of Streptomyces coelicolor A3(2) and sequence analysis of rrnA. Nucleic Acids Res 19, 4399-4403.

van Wezel, G. P., Woudt, L. P., Vervenne, R., Verdurmen, M. L. A., Vijgenboom, E. \& Bosch, L. (1994). Cloning and sequencing of the tuf genes of Streptomyces coelicolor A3(2). Biochim Biophys Acta 1219, 543-547.

van Wezel, G. P., Takano, E., Vijgenboom, E. \& Bosch, L. (1995) The tuf3 gene of Streptomyces coelicolor A3(2) encodes an inessential elongation factor $\mathrm{Tu}$ that is apparently subject to positive stringent control. Microbiology 141, 2519-2528.

Yanisch-Perron, C., Vieira, J. \& Messing, J. (1985). Improved M13 phage cloning vectors and host strains: nucleotide sequences of the M13mp18 and pUC19 vectors. Gene 33, 103-119.

Zhang, Y., Yu, N.-Y. \& Spremulli, L. L. (1998). Mutational analysis of the roles of residues in Escherichia coli elongation factor Ts in the interaction with elongation factor Tu. J Biol Chem 273, $4556-4562$.

Received 8 March 1999; accepted 4 May 1999 